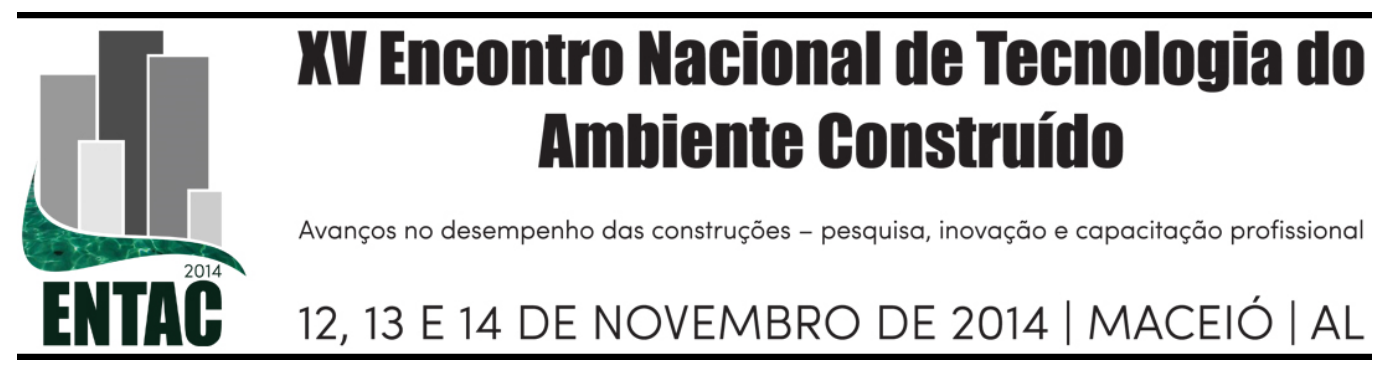

\title{
AVALIAÇÃO AUTOMÁTICA DE VALOR NO PROCESSO DE PROJETO DE HABITAÇÃO DE INTERESSE SOCIAL NO BRASIL
}

\author{
ANDRADE, Max (1); MATSUNANGA, Cristina (2) \\ (1) UFPE, e-mail: max.andrade@ufpe.br (1) UFPE, e-mail: criismnaga@gmail.com
}

\begin{abstract}
RESUMO
Este trabalho propõe um método de projeto que incorpora questão de valores a estimativa de custo, dentro de uma abordagem de custeio-meta e integrado ao Modelo da Informação da Construção (BIM). Este método de projeto incorpora mecanismos de avaliação do valor desejado associado ao custo, durante o processo de projeto de EHIS desenvolvidos sob a égide do programa federal Minha Casa Minha Vida (MCMV). A pesquisa mostra que existe uma real possibilidade de inserção de instrumentos metodológicos de projeto, que permita a avaliação de valores desejados, em tempo real, durante as ações projetuais. Esses valores podem contribuir para o redirecionamento de uma proposição projetual, possibilitando que as decisões dos projetistas sejam tomadas atendendo mais claramente aos desejos dos moradores, sem desconsiderar questões de custo, que são essenciais para a viabilidade de um empreendimento desse tipo.
\end{abstract}

Palavras-chave: Building Information Modeling, Valor desejado, Avaliação Automática, Custeio-meta.

\begin{abstract}
The method proposed in this paper addresses the management of values and the cost estimate, based on target costing approach, and integrated with Building Information Model (BIM). This design method must incorporate mechanisms of automated assessment associated with desired value at cost during the design process. The paper will try to show, in brief, that there is a real possibility of inclusion of methodological tools of design, allowing assessment of values in real time during the design actions.
\end{abstract}

Keywords: Building Information Modeling, Desired value, Automatic Assessment, Target Costing.

\section{INTRODUÇÃO}

A indústria da Arquitetura, Engenharia, Construção e Operação (AECO), no Brasil, no campo da habitação social vem passando por um processo de crescimento significativo nos últimos anos. No entanto, carece de métodos de concepção destinados a melhorar a qualidade das tipologias, tendo em conta a redução de custos. Confrontado com este problema, este trabalho apresenta uma pesquisa, em andamento, que propõe um método de projeto para ser usado na concepção de Empreendimentos Habitacionais de Interesse Social (EHIS). O método proposto neste trabalho trata da gestão de valores e estimativa de custo, em uma abordagem de custeio-meta, integrada ao Modelo da Informação da Construção (Building Information Modelling - BIM).

Para entender melhor esta abordagem, esta pesquisa iniciou-se definindo os conceitos de custo e valor e sua aplicação em habitação. De acordo com Silva (2008), custos são recursos aplicados na transformação dos ativos representados por gastos relativos à utilização de bens ou serviço aplicado na produção de outros bens ou serviços. Para Martins (1998) o custo pode ser entendido como um gasto monetário relativo à 
utilização de bens ou serviços utilizados na produção de outros bens. Ou seja, O custo é uma medida monetária dos sacrifícios financeiros que um indivíduo ou grupo de pessoas arcam a fim de atingir seus objetivos.

Já o conceito de valor, segundo Miron (2008) está intrinsecamente relacionado à satisfação para o cliente. Este autor, com base em Oliver (1980), infere que o julgamento da satisfação está sobre a influência do valor percebido pelo cliente, tanto na situação de pré-compra (valor desejado) quanto na situação de pós-compra (valor recebido). O valor recebido, por sua vez, está relacionado ao trade-off (ponderação) entre os benefícios recebidos pela aquisição do produto, ou serviço e os sacrifícios percebidos ao se confrontar com o custo destes, expresso pelo preço pago pelo mesmo.

A abordagem de custeio-meta (CM) é uma abordagem proveniente da indústria da manufatura que tem como objetivo gerenciar lucros (MONDEN, 1995) e resumidamente consiste em uma nova metodologia para se desenvolver produtos tendo em vista a relação entre o custo e o valor do produto pela perspectiva do cliente. Assim todas as informações são trabalhadas como dados que podem alimentar o processo de projeto ainda nas suas fases iniciais (JACOMIT; GRANJA, 2010).

Para colocar essa abordagem em prática, o método proposto neste trabalho trata da gestão de valores e estimativa de custo, em uma abordagem de custeio-meta, integrada ao Modelo da Informação da Construção. De acordo com Eastman et al. (2008), uma abordagem de projeto baseada no BIM pode proporcionar o uso da engenharia de valor no desenvolvimento de produtos da construção civil por meio de estimativas de custos e programação contínua, integradas na concepção do projeto. Com isso, facilita-se a tomada de decisão nas fases inicias de projeto, possibilitando uma avaliação do produto da construção sob uma abordagem de custeio-meta.

O método apresentado neste artigo busca incorporar mecanismos de avaliação do valor desejado associado ao custo monetário do empreendimento, já nos estágios iniciais do processo de projeto de EHIS desenvolvidos sob a égide do programa federal Minha Casa Minha Vida (MCMV). Para isso, foram estabelecidos alguns valores desejados pela população, a partir de pesquisas bibliográficas. Esses valores, com base em um método de monetarização, foram transformados em custos. Estes custos foram agregados à informações de projeto, associados ao modelo digital do edifício. Dessa forma, espera-se que seja possível, já nas etapas conceituais, compreender os custos (monetários) dos valores desejados pelos usuários.

\section{METODOLOGIA}

\subsection{Pesquisa Documental: definição do universo}

A primeira etapa da pesquisa, apresentada neste artigo, consistiu em uma análise crítica da produção arquitetônica e tecnologias construtivas de edifícios de EHIS, desenvolvidos nos últimos cinco anos na Região Metropolitana do Recife/ Brasil (RMR) e financiados pelo programa federal MCMV, nas faixas de renda 2 (renda de 4 a 6 salários mínimos) e 3 (renda de 7 a 10 salários mínimos). Para isso, foi realizada uma pesquisa documental.

Após o fichamento individual, as informações foram organizadas em uma tabela no Excel. Nesta tabela as respostas foram padronizadas e foram classificados em categorias de modo a facilitar a análise. Além das tabelas, alguns gráficos comparativos e correlações foram criados. 


\subsection{Pesquisa Documental: estudo de casos}

A partir de uma visão geral sobre o perfil da habitação, passou-se para a fase seguinte da pesquisa que constituiu da realização de estudos de casos com o objetivo de obter um levantamento mais completo, que confirmasse as informações catalogadas na fase anterior e detalhasse as informações relacionadas principalmente a tecnologia e processos construtivos.

Foram escolhidos como estudos de casos empreendimentos que ainda estão em construção e que são considerados como típicos, representativos da produção habitacional MCMV na RMR. Os estudos foram realizados através de visitas guiada à canteiros de obra, aplicação de questionários estruturados e entrevista com o arquiteto projetista, gerentes de obras e o engenheiro responsável pela obra.

\subsection{Pesquisa Experimental}

O passo seguinte está sendo o desenvolvimento de um método de projeto, apoiado em uma ferramenta computacional de autoria BIM (customizada) para o desenvolvimento de projetos arquitetônicos de EHIS, visando análise de custo e do valor percebido para o cliente, desde a fase de estudo preliminar. Os dados da pesquisa documental foram essenciais para definir o modo de operacionalização das variáveis e de inserção das informações em templates, que deverão ser utilizados pelos projetistas.

\section{A PESQUISA}

\subsection{Pesquisa Documental: catalogação de dados}

O Ministério das Cidades e a Caixa Econômica Federal estabelecem uma séria de regras descritas em cartilhas e especificações para o desenvolvimento de empreendimentos dentro do programa MCMV. Estas especificam o perfil do empreendimento, o porte, o dimensionamento das unidades habitacionais, o tipo de acabamento, as dimensões mínimas, as tecnologias recomendadas e uma série de exigências mínimas para a realização desses empreendimentos. Mesmo assim, cada empreendimento tem características particulares que os diferencia de outros. Essa diferenciação está vinculada à localização do empreendimento, as características do terreno, o perfil das construtoras, ao projeto arquitetônico, as tecnologias construtivas empregadas, à demanda local, questões regionais, entre outros fatores.

Buscando entender melhor as especificidades desse perfil de habitação na região de estudo delimitada por essa pesquisa o presente trabalho realizou a catalogação de 29 EHIS. Estes representam um total de 18.090 unidades habitacionais. Este valor representa cerca de $70 \%$ do total de empreendimentos realizados pelo programa MCMV na RMR, durante o período pesquisado. (Segundo dados do Ministério das Cidades este valor é de 26.039 unidades habitacionais, entre 2009/2014.) Com esse levantamento se obteve um catálogo representativo do perfil dos empreendimentos habitacionais que estão sendo lançadas na RMR. Entre os dados catalogados constam-se de dimensionamentos dos empreendimentos, perfil da implantação, características tipológicas, tecnologias construtivas empregadas, etc.

Entre as características dos empreendimentos pesquisados observa-se que os mesmos reproduzem o perfil programático de empreendimentos voltados para uma população de maior renda: são todos eles condomínios fechados compreendidos por empreendimentos de médio porte (47\% dos casos são edifícios constituídos por 1 a 10 blocos de apartamentos; $70 \%$ dos empreendimentos são constituídos por até 500 apartamentos); 
todos os empreendimentos possuem 1 vaga de automóvel por apartamento e possuem áreas de lazer, com diversos equipamentos de apoio; são condomínios constituídos por blocos de 4 pavimentos (70\%), mas nos últimos anos, por conta de uma mudança tecnológica, estão sendo substituídos por blocos de 7 a 8 pavimentos (30\%) com elevador; são empreendimentos com áreas que variam entre 41 a $50 \mathrm{~m} 2$; e são constituídos, em sua grande maioria, por apartamentos de 2 dormitórios $(74 \%$ empreendimentos). Quanto às tecnologias construtivas utilizadas destacam-se as seguintes tecnologias: sistema de paredes de concreto in loco, estrutura de concreto armado com pilar e viga e a alvenaria de bloco estrutural de concreto.

\subsection{Pesquisa Documental: estudo de casos}

Com relação ao processo de tomada de decisões para a realização dos empreendimentos, o que se tem observado, a partir dos estudos de casos, é que afora as restrições estabelecidas pelo Ministério das Cidades e pela Caixa Econômica Federal, a equipe de vendas das incorporadoras é o principal instrumento que define o porte, a localização dos empreendimentos, e questões programáticas, como por exemplo, a existência de piscinas, quadras de esporte, etc. O custo vai ser definido com base no uso de uma tecnologia construtiva ou outra. O papel dos projetistas é secundário, assim como questões de desempenho e de valor, que poderiam estar melhor agregada ao projeto dos empreendimentos.

\subsection{Pesquisa Experimental}

Esta fase da pesquisa, em andamento, analisa as questões relacionadas com o valor e o custo da habitação e propõe metodologia para o desenvolvimento de projetos de habitação apoiados pela avaliação de valores e sustentados no conceito BIM.

\subsection{Valor Desejado da Habitação de Interesse Social no Brasil}

Uma das abordagens mais comumente discutidas em habitação de interesse social, no Brasil, trata do custo da produção e manutenção dos EHIS. Essas, pouco consideram questões de valor associadas ao custo da habitação. Abordagens que considerem o valor são, geralmente, empíricas e pouco contribuem para a qualidade da solução, sendo difícil de levar à cabo durante o desenvolvimento de projetos. Por outro lado, autores como Maslow (1998) mostram que uma abordagem baseada em valores, a partir da teoria da hierarquia das necessidades, deveria se constituir um elemento chave em qualquer projeto de edificação.

A partir de análises de trabalhos que avaliam o valor desejado em EHIS no Brasil foi possível identificar valores mais relevantes e que poderiam ser quantificados e introduzidos em processos de projeto de EHIS. Entre estes trabalhos destacam-se: Granja et al. (2009) e Formoso et al. (2011). Embora esses trabalhos representem realidades culturas, econômicas e sociais diferentes da realidade da presente pesquisa, estas serviram como referências de eventuais valores desejados referenciados nesta pesquisa.

Na prática projetual, o que se observou, nos estudos de casos, é que os valores presentes nos programas de EHIS na RMR são definidos de maneira empírica, a partir de análises de mercado estabelecidas pelos Corretores de Imóveis. Estes profissionais, a partir de conversas com o público e de um conhecimento do que se vende e o que não se vende, indicam para as empresas incorporadoras alguns dos principais "valores" que devem ser considerados em novos empreendimentos de EHIS. 
Visando ter um referencial de valores este pesquisa utiliza uma base de dados de pesquisas realizadas com populações advindas de realidades sócios culturais diferentes, porém com um público de faixa econômica parecida. Assim, utilizou-se como valores de referência aquelas levantados por Granja et al. (2009) e Formoso et al. (2011). Para Granja et al. (2009) os valores foram agrupados em cinco categorias: Perspectiva Financeira, Percepção Sócio-espacial, Valores Culturais, Qualidade do Ambiente Interno e Qualidade Espacial. Para Formoso et al. (2011) a abordagem envolveu questões como: habitabilidade, funcionalidade, segurança, aparência, acessibilidade a transporte e equipamentos urbanos, etc.

A partir da análise dos valores considerados mais importantes nas duas pesquisas acima supracitada e com base nos valores que poderiam ser melhor quantificáveis e transformados em dados de projeto, esta pesquisa se propôs a trabalhar com os seguintes valores desejados: (1) Baixo Custo de Manutenção; (2) Segurança/ Privacidade; (3) Acessibilidade; (4) Existência de Área de laser; (5) Custo das Unidades; (6) Qualidade Acústica; (7) Qualidade Térmica; (8) Conforto Ambiental; (9) Tamanho das unidades.

Com base nas análises empreendidas na pesquisa documental e em autores como Eastman (2009), Eastman et al. (2011) esta pesquisa propõe um método de projeto que avalia o valor desejado da solução arquitetônica, a partir de informações de custo do empreendimento, já nas etapas inicias do processo de projeto. Com isso, é possível estabelecer uma correlação entre o custo das decisões projetuais, e o valor desejado pelo morador.

Estabelecidos os valores desejados, procurou-se identifica categorias de custo existentes em um empreendimento MCMV que pudessem estar relacionados com esses valores. Entre as categorias de custos identificados destacam-se: custo absoluto e custo comparado. $O$ custo absoluto se refere a um custo que é padrão para todas as unidades habitacionais, dentro de um empreendimento. Este custo, que é fixo e igual para todas as unidades habitacionais, varia em função da característica do empreendimento. $\mathrm{O}$ custo relativo se refere ao custo agregado ao apartamento em virtude de características específicas da unidade habitacional. Este pode variar em função da localização e disposição da unidade em relação ao conjunto habitacional. Também pode variar em função as tecnologias usadas e das soluções empreendidas de projeto.

Para o cálculo do custo também se levou em consideração a vida útil do empreendimento. Para o cálculo da Vida Útil, utilizou-se como referência a European Organization for Technical Aprovals (CIB Report Publication 294, 2004 apud ASSOCIAÇÃO BRASILEIRA DE NORMAS TÉCNICAS, 2008). Esta organização considera a Vida Útil de um empreendimento habitacional de 50 anos. Logo, para o cálculo do custo do valor desejado deve-se considerar o custo, mensal ou anual, multiplicado pelo tempo de vida útil do empreendimento, considerado por esta pesquisa como de 50 anos.

No quadro abaixo (Quadro 1) são apresentados os valores desejados, considerados como os mais importantes nesta pesquisa, a descrição resumida desses valores e os referenciais de custos, obtidos na pesquisa documental deste trabalho. A partir de cada um desses valores, obteve-se os custos agregados a cada um desses valores. Nos itens à seguir, são apresentados suscintamente como se obteve dois desses valores.

\section{Quadro 1 - Valores desejados em projetos habitacionais MCMV}




\begin{tabular}{|c|c|c|c|}
\hline 1 & $\begin{array}{l}\text { Baixo Custo de } \\
\text { Manutenção }\end{array}$ & Relação unidade/conjunto & $\begin{array}{l}\text { Referência: } 380 \text { unidades } \\
\text { Acima - reduzir custo } \\
\text { Abaixo - aumentar custo }\end{array}$ \\
\hline 2 & $\begin{array}{l}\text { Existência de } \\
\text { área de lazer }\end{array}$ & $\begin{array}{l}\text { Relação área de lazer/ número de } \\
\text { unidades }\end{array}$ & $\begin{array}{l}\text { Referência: } 0,5 \mathrm{~m}^{2} / \text { Unid. } \\
\text { Acima - aumentar custo } \\
\text { Abaixo - reduzir custo }\end{array}$ \\
\hline 3 & $\begin{array}{l}\text { Conforto } \\
\text { Ambiental }\end{array}$ & $\begin{array}{l}\text { Custo Total da } \\
\text { Construção/densidade do } \\
\text { empreendimento }\end{array}$ & $\begin{array}{l}\text { Densidade de referência: } 55 \mathrm{~m} 2 \\
\text { Acima - aumentar conforto } \\
\text { Abaixo - reduzir conforto }\end{array}$ \\
\hline 4 & Custo do $\mathrm{m} 2$ & $\begin{array}{l}\text { Valor em Reais obtidos a partir do } \\
\text { custo total da construção pelo } \\
\text { número de apartamentos. }\end{array}$ & $\begin{array}{l}\text { Quando o valor do m2 estiver acima do valor de } \\
\text { referência o empreendimento terá um custo maior, } \\
\text { e vice-versa. }\end{array}$ \\
\hline 5 & $\begin{array}{l}\text { Maior } \\
\text { Segurança/ } \\
\text { Privacidade }\end{array}$ & $\begin{array}{l}\text { Visibilidade/ segurança dos } \\
\text { apartamentos no Térreo }\end{array}$ & $\begin{array}{l}\text { Térreo - Custo de barreira vegetal }(\mathrm{R} \$) / \text { Custo } \\
\text { grade } \\
\text { Nos demais andares o custo é zero }\end{array}$ \\
\hline 6 & $\begin{array}{c}\text { Boa } \\
\text { Acessibilidade }\end{array}$ & $\begin{array}{l}\text { Acessibilidade aos apartamentos - } \\
\text { térreo - boa, demais andares ruins. }\end{array}$ & $\begin{array}{c}\text { No térreo a acessibilidade é boa e o custo é zero. } \\
\text { Nos demais deve-se acrescentar o custo elevador/ } \\
\text { número de unidades por bloco (não incluir o } \\
\text { térreo) }\end{array}$ \\
\hline 7 & $\begin{array}{l}\text { Qualidade } \\
\text { acústica }\end{array}$ & $\begin{array}{l}\text { Custo de reparação. Em função da } \\
\text { localização das unidades em } \\
\text { relação à fontes de ruído. }\end{array}$ & $\begin{array}{c}\text { Bom - custo 0 } \\
\text { Regular }-\mathrm{R} \$ \\
\text { Ruim }-\mathrm{R} \$ \text { (tratamento acústico mais severo) }\end{array}$ \\
\hline 8 & $\begin{array}{l}\text { Qualidade } \\
\text { térmica }\end{array}$ & Custo para refrigeração. & Referência Leste/ventilação cruzada. \\
\hline
\end{tabular}

Fonte: Autores

\subsubsection{Valor Desejado: menor valor do condomínio}

Um dos valores mais desejados pela população é que o empreendimento tenha um baixo custo de manutenção, o que causa uma sensação de conforto. Este custo está diretamente associado ao custo do condomínio. Para o cálculo do custo do condomínio levou-se em consideração a quantidade de unidades habitacionais por empreendimento. Empreendimentos com muitas unidades, o custo do condomínio é, teoricamente, reduzido. Inicialmente criou-se um custo de referência. Este foi obtido com base no número médio de apartamentos por condomínio pesquisado. O número foi de 330 unidades por empreendimento. $(\mathrm{O}$ valor monetário de referência do condomínio foi obtido com base nos custos de condomínio praticados em empreendimentos MCMV na RMR, já em funcionamento (fev/2014).) Este custo foi de $\mathrm{R} \$ 158,00$. Um empreendimento com 295 unidades de apartamentos, por exemplo, o custo do condomínio seria de $\mathrm{R} \$ 176,00$, ou seja, um aumento de $\mathrm{R} \$ 18,00$ em relação ao custobase. Para se obter o custo do valor desejado multiplica-se o custo da diferença entre o custo de referência e o custo real e multiplica este valor pela Vida Útil do edifício (50 anos/600 meses), obtém-se o custo de manutenção do empreendimento (R \$ 10.800,00).

Embora o custo de manutenção se reduza com o aumento do número de unidades do empreendimento, outros valores desejados, como o conforto ambiental está associado à redução da densidade do empreendimento. Portanto, serve como medida de compensação.

\subsubsection{Valor Desejado: qualidade térmica}


Outro exemplo de valor desejado que foi quantificado nesta pesquisa foi a qualidade térmica. Este está associada à qualidade térmica de um apartamento em função da sua disposição em relação ao Norte e em relação à existência ou não de ventilação cruzada. Assim, um apartamento (A), que possui a orientação principal Leste e Ventilação Cruzada, possui (em tese) uma melhor qualidade térmica de que um apartamento cuja orientação principal é Oeste e não tem ventilação cruzada.

Para quantificar, em termos monetários, o custo da qualidade térmica dos apartamentos, em função da localização realizou-se o seguinte procedimento: construiu-se um modelo digital de um bloco de apartamento padrão (modelo fornecido pelo manual do MCMV Figura ); Fez-se os estudos das cargas térmicas e da quantidade de calor necessário para a refrigeração de quatro fachadas (Norte, Sul, Leste e Oeste); A carga térmica da melhor posição é considerada com o valor de referência; A partir daí são avaliadas as cargas das demais posições e estabelecida a quantidade de BTUs necessário para se chegar ao valor de referência (Melhor posição).
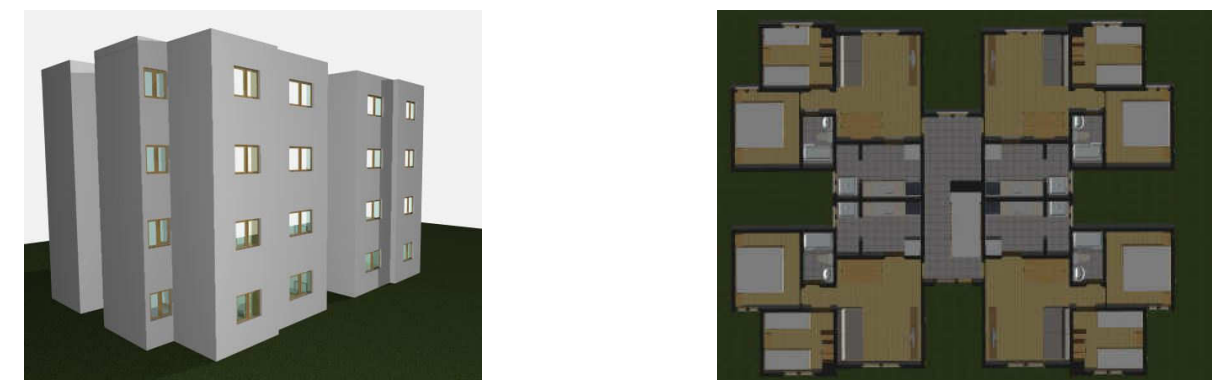

Figura 1 Modelo padrão MCMV proposto em manual (modelo digital do MCMV modelo no ArchiCAD da Graphisoft).

A partir do modelo 3D do edifício realizou-se o estudo da carga térmica. Para isso utilizou-se a ferramenta Avaliação Ecodesigner (que é uma extensão do ArchiCAD da Graphisoft). Foram consideradas duas situações de uso dos apartamentos, para o cálculo energético: o apartamento com as janelas fechadas e condicionadas a uma temperatura de 24 graus e os apartamentos com as janelas abertas, considerando a ventilação natural e a quantidade de calor que incide na fachada. Para esta última opção considerou-se como temperatura de referência 25 graus. Para cada situação foram consideradas as 4 posições geográficas, Norte, Sul, Leste e Oeste.

Os resultados mostram que a melhor posição é a fachada Norte. Para esta fachada seria necessário $10.428 \mathrm{KW}$ /ano para que o ambiente permanecesse com uma temperatura de 25 graus. Para as demais fachadas a quantidade de energia necessária para se obter a mesma temperatura precisaria de ser de 10.625(Leste), 10.651(Sul) e 10.864 (Oeste) KW/ano.

Para se obter o custo total de energia considerou-se como valor de referência a quantidade de energia em Quilowatts/Ano que cada uma das outras três fachadas, precisaria de gerar para se obter o mesmo valor da fachada de referência (Fachada Norte). Assim foi possível obter a quantidade de Energia necessária para que as demais fachadas do empreendimento tivessem a mesma qualidade térmica da fachada Norte (melhor fachada). Para as fachadas Leste, Sul e Oeste seriam necessárias as seguintes cargas térmicas para se chegar ao mesmo padrão de aquecimento da fachada Norte: 197 Quilowatts/Ano (fachada leste); 223 Quilowatts/Ano (fachada sul); e, 436 Quilowatts/Ano (fachada oeste). Considerando como valor de referência do Quilowatts/hora de R \$ 0,50, ter-se-ia como custo agregado às demais fachadas leste, sul 
e oeste, de $R \$ 98,50, R \$ 111,50$ e 218 reais por ano. Considerando a vida útil do empreendimento tem-se que os custos agregados são de respectivamente de R $\$ 4.925$, $\mathrm{R} \$ 5.575$ e $\mathrm{R} \$ 10.900$.

\subsubsection{Custo Final do Imóvel}

A partir das considerações abreviadamente apresentadas acima se chegou a uma relação de Custos dos Valores Desejados e impactos desses no Custo Final do Imóvel (CFI). O CFI é a soma do Custo Total da Construção (CTC) do Imóvel acrescido do Custo do Valor Desejado (CVD), que é a soma dos custos dos valores desejados nesta pesquisa.

Para a obtenção do CVD foram utilizados como parâmetros dados obtidos na pesquisa documental, além de informações da cartilha e especificações do programa MCMV. Para esta etapa da pesquisa esses valores foram formulados de maneira empírica, sem uma calibração (o que deverá ocorrer na etapa seguinte da pesquisa).

Para o Custo Total da Construção (CTC) foram levadas em consideração, as tecnologias construtivas utilizadas. As tecnologias identificadas nos estudos de casos foram: paredes de concreto in loco, estrutura de concreto armado com pilar e viga e a alvenaria de bloco estrutural de concreto. Para cada uma dessas tecnologias foram estudados os componentes que tinham maior peso na composição de preço da construção.

Acredita-se que ao se obter o CVD e o CTC, a partir de soluções de projeto propostas ainda na fase de estudo preliminar, será possível com que projetistas e construtores avaliem, com muito mais precisão, os custos das decisões arquitetônicas em projetos de EHIS e os impactos desses custos no CFI.

Para que os resultados da pesquisa fossem considerados como satisfatórios seria necessário que os mesmos estivessem agregados às ferramentas de avaliação automática, utilizadas pelos projetistas durante o processo de projeto. Dessa maneira, seria possível que os valores pudessem ser mensurados automaticamente, em virtude das decisões projetuais.

\subsection{Implementação em software de autoria BIM}

$\mathrm{Na}$ etapa final, desta pesquisa está sendo proposto um processo metodológico que permita com que o CVD seja obtido juntamente com o lançamento do estudo preliminar de uma solução de projeto. A ideia é que o CVD esteja agregado em templates de softwares de autoria BIM. Estes templates deverão ser utilizados pelos projetistas já durante as fases iniciais do processo de projeto. Estes templates também incorporarão informações sobre as tecnologias construtivas utilizadas neste tipo de empreendimento, e identificadas na pesquisa documental. Estes templates, portanto, conterão as famílias de objetos típicas dessas tecnologias.

É importante destacar que os CVD precisam ser transformados em valores monetários e precisam estar "pendurados" em templates, de modo que possam ser resgatados, em função da característica do projeto. Para a análise final de uma proposta o modelo deverá fornecer informações do CVD e do CTC. Este último deverá ser obtido (em termos de estimativa) a partir das tabelas dos quantitativos obtidos de modo automático nos templates.

Com relação à inserção de informações para a obtenção dos valores procurou-se minimizar os trabalhos dos projetistas, de modo que os mesmos tivessem o mínimo de trabalho, de modo a comprometer ao mínimo o processo projetual. Para isso, limitou a inserção da informação dos valores desejados a um único sistema do edifício: Zona (As 
zonas, de acordo com a Graphisoft 2013, são unidades espaciais do projeto).

A partir de dados obtidos diretamente das zonas, é possível extrair os custos dos principais valores considerados na pesquisa. Essas informações de zonas estão sendo inseridas também nos templates de projeto dentro de um software de autoria BIM. O software que esta sendo usado é o ArchiCAD da Graphisoft. Dentro desses templates estão sendo criadas tabelas automáticas, que fornecem esses índices.

$\mathrm{Na}$ zona são associadas informações sobre a localização geográfica das unidades de apartamentos, a área, o pavimento, questões acústicas, etc. Essas informações são posicionadas em localizações estratégicas dentro de tabelas.

Ao modelar as zonas das unidades habitacionais com as informações dos valores desejados, o projetista exporta a tabela gerada automaticamente, para o software Excel da Microsoft. Neste último, esta sendo criado um Macro, com informações sobre os valores de referência pesquisados neste trabalho. Assim, ao abrir o Macro nas tabelas exportadas do software de autoria BIM é possível obter de maneira automatizada os resultados tanto do CVD e quanto do CTC.

O que se espera, por fim, é durante o desenvolvimento da proposta de uma solução arquitetônica de HIS, já no estudo preliminar, seja possível obter o CVD e o CTC da solução arquitetônica proposta. Desse modo, será fácil e rápido identificar os impactos das alterações espaciais no CVD e CTC. Com isso também será possível empreender ações de projeto que valorizem mais os valores desejados pelos moradores e identificar como esses valores podem interferir no custo do empreendimento.

A obtenção do CTC está sendo obtida também de maneira automática, a partir da definição daquelas famílias de componentes que têm maior impacto no custo do empreendimento. Essas famílias estão agregadas aos templates, que, por sua vez, reproduzem as tecnologias construtivas utilizadas nesse tipo de empreendimento.

\section{LIMITAÇÕES}

Como o foco principal do estudo está na proposição de um método de projeto, baseado o uso de informações de valores desejados "pendurados" nos modelos digitais de edifícios para a obtenção do CVD e CTC, é necessário que os valores considerados na pesquisa sejam validados regionalmente. Ressalta-se que esses valores foram considerados apenas como meio de validação de um método de trabalho. Na prática, precisar-se-iam desenvolver pesquisas em valor, dentro do MCMV na RMR.

Outra questão que não foi considerado neste trabalho, mas que deve ser visto como de extrema importância foi à calibração dos valores monetários considerados nesta pesquisa. Esses valores foram obtidos de maneira empírica, a partir de dados analíticos dos conjuntos habitacionais estudados neste trabalho e precisariam ser validados.

Por fim, entende-se que para comprovar a eficiência do método proposto nesta pesquisa seriam necessários alguns testes de projeto, com estudos de análise de protocolos. Com isso, seria possível efetivamente validar a eficiência do método.

\section{CONCLUSÕES}

O que se conclui com esta pesquisa, embora com resultados ainda muito preliminares, é que existe uma real possibilidade de inserção de instrumentos metodológicos de projeto, que permita a avaliação de valores desejados, em tempo real, durante as ações projetuais. Esses valores podem contribuir para o redirecionamento de uma proposição 
projetual, possibilitando que as decisões dos projetistas sejam tomadas atendendo mais claramente os desejos dos moradores, sem desconsiderar questões de custo, que são essenciais para a viabilidade de um empreendimento desse tipo. Mas, para isso a incorporação e extração de informações no modelo digital do edifício aparecem como instrumento essencial para a viabilização do método.

\section{AGRADECIMENTOS}

Às construtoras e escritórios de projeto que participaram da pesquisa.

\section{REFERÊNCIAS}

ASSOCIAÇÃO BRASILEIRA DE NORMAS TÉCNICAS. NBR 15575-1: Edifícios habitacionais de até cinco pavimentos - Desempenho - Parte 1. Rio de Janeiro: ABNT, 2008.

EASTMAN, C. Automated assessment of early concept designs. Architectural Design, n. 199, p. 52-57, May-Jun. 2009. 2009.

EASTMAN, C.; TEICHOLZ, P.; SACKS, R.; LISTON, K. BIM Handbook: A guide to Building Information Modeling for owners, managers, designers, engineers, and contractors. 2nd ed, Hoboken, New Jersey: John Wiley \& Sons. 2011.

FORMOSO, C.; BONATTO, F. ; MIRON, L. Avaliação de empreendimentos habitacionais de interesse social com base na hierarquia de valor percebido pelo usuário. Ambiente Construído (Online), v. 11, p. 67-83. 2011.

GRANJA, A. D., KOWALTOWSKI, D. C. C. K.; PINA, S. A. M. G.; FONTANINI, P. S. P.; BARROS, L. D. F.; PAOLI, D. D.; JACOMIT, A. M.; MAÇANS, R. M. R.. A natureza do valor desejado na habitação social. Revista Ambiente Construído, v.9, n.2, p. 87-103. Abr/Jun de 2009. 2009.

GRAPHISOFT. Help Center, 2014.

JACOMIT, A. M; GRANJA, A. D. Análise crítica da aplicação do custeio-meta no desenvolvimento de empreendimentos de habitação social. Revista Ambiente Construído, v. 10, n 1, p. 69-88 Jan/Mar 2010.

MARTINS, Eliseu. Contabilidade de Custos. $6^{\circ}$ Ed. São Paulo: Atlas, 1998.

MASLOW, A. Maslow on Management.New York: Wiley. 312p. 1998.

MIRON, L. I. G. Gerenciamento dos Requisitos dos Clientes de Empreendimentos Habitacionais de Interesse Social: proposta para o programa integrado entrada da cidade em Porto Alegre, RS. 351 f. Porto Alegre. 2008. Tese (Doutorado em Engenharia Civil) - Escola de Engenharia, Universidade Federal do Rio Grande do Sul, Porto Alegre, 2008.

MONDEN, Y. Target Costing and Kaizen Costing: portland, Oregon, USA: Productivity Press, 1995.

MORAIS, M.; GRANJA, A. D.; RUSCHEL, Regina Coeli; BIM e custeio-meta: uma análise em HIS, 11/2011, VII Simpósio Brasileiro de Gestão e Economia da Construção (SIBRAGEC),Vol. 1, pp.1-10, Belém, PA, Brasil, 2011

SILVA, Benedito Albuquerque da. Custos e Estratégias de Gestão. Apostilado de pósgraduação, 2008. 